\title{
Design and Implementation of Electronic Textbook Resources based on Cloud Service
}

\author{
Biao Dong ${ }^{1, \text { a }}$ \\ ${ }^{1}$ Nanjing Institute of Industry Technology, Nanjing, China \\ adongb@niit.edu.cn
}

Keywords: e-textbook; service; resource

\begin{abstract}
Electronic textbook(E-textbook) resources include reading resources in the field of digital reading, and learning resources in the field of digital learning. The emergence of cloud service efficiently supports the construction of the e-textbook resource management. Cloud service is a suitable architecture for e-textbook resources model. This paper designs ETRCS, An e-textbook resources management model based on cloud service, which includes three aspects, the two-stage conversion process, the resources format conversion, and the relationships between e-textbook content objects. ETRCS resources addressing is composed of several addressing iterative layers, namely hierarchical model of ETRCS. We present an application architecture model of ETRCS, which is based on the hierarchical model of ETRCS.
\end{abstract}

\section{Introduction}

With the evolution of information media, the era of e-textbook is coming. Compared to paper book, e-textbook achieves a qualitative leap, plays a role in connecting digital reading and learning. E-textbook provides support for new learning mode under the digital environment. Specific performance is as follows: e-textbook supports the differentiated class teaching, the collaborative group learning, the personal interest expansion learning, the online interactive learning. And the single, uniformly edited content resources will evolve into personal miniature knowledge spaces which are based on personalized contents, learning tools and services. Digital reading and digital learning are two respective professional fields, the two fields are separated but linked together. They are different to each other in resource form, content format, packaging mode, organization structure, and application situation. E-textbook resources mainly include both reading resources in the field of digital reading and learning resources in the field of digital learning. The resources integrate the dual attributes of the two fields[1].

Users are demanding more and better service, delivered through every conceivable channel. Cloud service as a service establishes the industry standard and user self-service. Users need not be aware computer operating system, middleware, application and communication networks. Only according to their own needs and through the networks, the Users use a variety of terminal equipments, such as fixed, mobile, and all kinds of home terminal devices, and select the appropriate services, such as computing, storage, learning, entertainment, interaction, etc.

E-textbook resource cloud service refers to a process. In this process, cloud service providers meet user's demand for the e-textbook resources. The demand of e-textbook resources involves in design, development, transmission, application, evaluation, and other corresponding services. At the same time, users need not pay attention to technical implementation and support environment related to e-textbook resource services. E-textbook resource cloud service has good scalability and rapid scalability, so, e-textbook resources can be obtained at any time as needed.

This paper presents the model and system design that tailor ETRCS to e-textbook resource cloud service environment. Specifically, it makes the following primary contributions.

(1) Propose e-textbook resource model based on cloud service, and define resource object relations of e-textbook.

(2) Design and implement the hierarchical model and application architecture model of ETRCS. 


\section{ETRCS model}

In cloud service environment, ETRCS is used to describe the form of the e-textbook resources, the content conversion between e-textbooks resources and other resources. It's the foundation of the e-textbook domain characteristics. E-textbook resources mainly include reading resources in the field of digital reading, and learning resources in the field of digital learning. E-textbook should adopt a relatively stable packaging, to achieve structured, object-oriented features. Its content unit is a package which contains material and can be regarded as a learning object. The content packages of e-textbook don't damage the underlying material resource format, but make logical level packaging on the underlying material format, and add additional information. So this design can be done to maximize compatibility. Resource object relations of e-textbook are shown as follows. Firstly, how can the paper resources shift into the digital resources? Secondly, how can the resources with a single file structure shift into the structured content objects which have the characteristics of learning objects and are based on the content packages? ETRCS mainly includes three aspects as follows.

(1) A two-stage conversion process for converting the organizational structure of the e-textbook. In the digital reading resources, the EPUB packaging format has the characteristics of good compatibility, simple, high efficiency, and wide application[2]. We adopt the EPUB standard content package structure and the general ZIP packet encapsulation mode as a basic mode of packing e-textbook. The IMS-CP packaging structure has been widely used in the digital learning resources, and has been recommended by the subsequent IMS-CP, SCORM and other standards[3,4]. The IMS-CP content packaging structure always comes with the e-textbook content packaging attached. Therefore, the relationship between electronic textbooks and other resources can form a two-stage conversion relationship; that is, the conversion of the digital reading resources mediated by the EPUB content packaging is the first conversion; the conversion of the digital learning resources mediated by the IMS-CP content packaging is the second conversion. By the two-stage conversion process, the digital reading resources and the digital learning resources are uniformly packed into the e-textbook. This design provides great a convenience at the metadata level. Metadata information that is carried by the EPUB content package is directly converted into the cover metadata of e-textbook. The cover metadata are used for search engines to search the whole e-textbook. The LOM metadata of the IMS-CP package are simplified into the metadata of the e-textbook content objects[5].

(2) E-textbook resources format conversion process. Ordinary paper books are digitally processed into digital reading resources, which resource form is a single digital file. According to the subject content, the resources are cut into small theme file blocks, which are stored in the file repository and used as the digital reading materials. According to the information model of the e-textbook, we logically reorganize the file resources to form the e-textbook content objects, which are stored in a content object repository. According to the content packaging way of e-textbook, we extract the digital reading resources from a file repository, and extract digital learning resources from a content object repository. We combine the two methods to form an e-textbook. The above conversion process can not only meet the content format of the e-textbooks and the digital reading resources at minimal cost, but also has a lot of advantages of the EPUB content packaging and the IMS-CP content packaging.

(3) The relationships between e-textbook content objects. E-textbook content materials can include pictures, texts, video, audio, program body and other documents. After format conversion and packaging, the materials form the content packages, which have the learning object morphological features, to achieve structural characteristic of e-textbook. From the perspective of the interaction between learning objects, these relationships can be divided into three specific relationships, such as aggregation, coupling, and separation. The two learning objects which are associated with the aggregation relationship are completely integrated into a content object, and their underlying contents are fused into one package. The coupling relationship refers to the mashup relation between the content objects, there is a certain correlation between the content objects, but their underlying contents aren't fused into one package. Depending on the degree of correlation, the coupling relationship can be subdivided into loose coupling and tight coupling. The two learning objects, 
which are associated with the separation relationship, are completely broken down into two separate parts which have their respective closure packages. The separation and the aggregation are the reverse operations with each other.

\section{Hierarchical model and application architecture model of ETRCS}

Hierarchical model. Hierarchical model of ETRCS is extended from the layered iteration model of Internet resources, it has hierarchical iterative rules. ETRCS resources addressing is composed of several addressing iterative layers, the output of each addressing layer can be considered as the input of the next addressing layer. For each layer of resource addressing system, it does not need to understand all the underlying logic, only need to understand the interface of the adjacent layers. This reduces the coupling degree between the resource addressing systems, and makes us think more about the implementation of the subsystem rather than the interactions between the interval subsystems. The realization way of each layer of resource addressing subsystem can be different. We can also use the same way to complete the addressing function in the different layers. But the addressing subsystem still belongs to different layers in the logic.

ETRCS's resource name can not be used directly as the input of the resource addressing subsystems, the appropriate conversion operations are required to remove the possible dispersibility and unknowability. In the hierarchical model of ETRCS, e-textbook resource names are subdivided into the original resource names and the converted resource name. Resource information, which is needed to convert the resource name from the original resource name to the converted resource name, can be provided by the output of the upper layer. Similarly, the resource address outputted by the layer may also be used as the conversion information of the next layer.

Application architecture model. We present an application architecture model of ETRCS, which is based on the hierarchical model of ETRCS. The model analyzes and summarizes the relationships between the ETRCS resource addressing systems, and divides the ETRCS resource addressing system into five layers, such as standard layer, coding layer, discovery layer, information layer, and physical address layer. The application architecture model of ETRCS is shown in Fig.1.

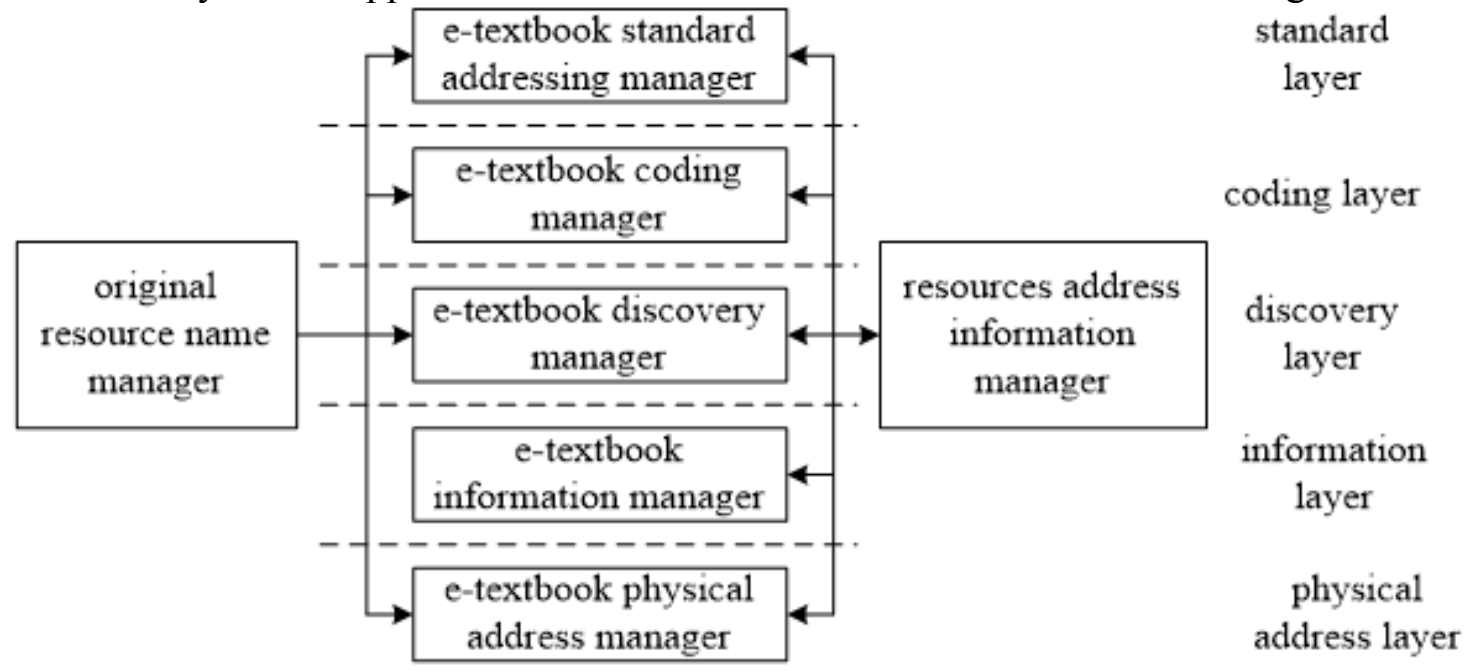

Fig.1. Application architecture model of ETRCS

Standard layer may be an iterative form of multiple resource addressing layers. Therefore, the converted information of the first resource addressing layer is known as the default. The converted information of the other resource addressing layers is provided by the resources address information, which is outputted by the upper resource addressing layer. Because there's diversity in the hierarchical structure of the next layer of e-textbook coding, therefore, there will be such a situation, that is, the different sub-types of e-textbook codings correspond to the same standard identification codes. This also results in the dispersive and unknown hierarchical structure of the standard identification codes. So the original resource name of the standard layer is a combination of standard identification codes and e-textbook resource codings. 
Coding layer locates on the second layer, its original resource names are e-textbook codings. E-textbook coding has its own particularity. In contrast to the coding addressing system of the layer, the coding addressing system of the layer is of dispersibility. In terms of the resource addressing system based on a hierarchical addressing algorithm, its hierarchical structure is of unknowability. According to e-textbook resource address information provided by the upper layer, the layer needs to convert the original resource names into the converted resource names. Then, by the e-textbooks coding and addressing system, the layer completes the process of addressing from the e-textbook coding to the related resource address. The resource address information, which is outputted by the layer's addressing system, is the address information of the specific resources associated with e-textbook coding, and is the resource address information required by the fourth layer, namely information layer. The resource address information may also be the address information associated with e-textbook information discovery services, and is the resource address information required by the third layer, namely discovery layer.

Discovery layer locates on the third layer, its original resource names are the same e-textbook codings. The layer completes the process of addressing from the e-textbook coding to the related resource address, which can participate in the addressing of the e-textbook discovery system.

Information layer locates on the fourth layer, its original resource names can be null. The layer receives resource address information which is outputted by the second and third layer. These resource addresses are equivalent to indirect resource addresses, which can be directly used as the converted resource names of the layer to participate in addressing. Resource address information, which is outputted from the layer to the next layer, is IP address.

Physical address layer is located at the bottom to complete addressing from IP address to physical address.

\section{Conclusions}

In this paper, we propose the e-textbook resource model based on cloud service. ETRCS model is defined in a service-oriented e-textbook resource management environment. We illustrates a conclusion through hierarchical model and application architecture model of ETRCS. The results show that ETRCS can easily be constructed.

\section{Acknowledgements}

This work was sponsored by Qing Lan Project(Jiangsu province, China), 2014 annual project of institute of modern educational technology, Jiangsu research institute of education sciences(NO. 2014-R-31996, modeling and implementation of the electronic textbook based on mobile terminal equipments).

\section{References}

[1] J.Y.Lai, K.R.Ulhas: Electronic Library, The, Vol.30(3) (2012), p.321-338.

[2] J.Buse: EPUB from the Ground Up: A Hands-on Guide to EPUB 2 and EPUB 3(McGraw-Hill Osborne Media, 2013).

[3] Á.Del Blanco, E.J.Marchiori, J.Torrente, et al.: Computer Standards \& Interfaces, Vol.36(1) (2013), p.178-187.

[4] E.Kurilovas, S.Serikoviene: Technological and Economic Development of Economy, Vol.19(4) (2013), p.706-723.

[5] B.Jebali, R.Farhat: Ontology-based semantic metadata extraction approach(ICEESA, 2013). 